\title{
¿Prevención de convulsiones con sulfato de magnesio en preeclampsia?
}

\begin{abstract}
Caso clínico:
Un Residente de primer año recibe en su guardia a una embarazada de 35 años cursando su semana 34 de gestación. Es primigesta y consulta por cefalea. Al examen físico se le detecta una tensión arterial (TA) de 150/95 mm Hg e hiperreflexia. La proteinuria es de ++. La paciente queda en observación en reposo absoluto y a las seis horas la TA se mantiene en los valores iniciales y la proteinuria persiste. El residente decide internarla con diagnóstico de preeclampsia moderada. El residente mayor acuerda con su conducta, pero en el pase de la mañana el Jefe de Residentes le recrimina no haber indicado Sulfato de Magnesio (SM) u otro anticonvulsivante para prevenir la convulsión eclámptica. El Residente sabe que existen algunos estudios acerca de este tema, pero quiere verificar la evidencia disponible a fin de determinar la racionalidad de su conducta o la que le sugiere su Jefe.
\end{abstract}

\section{Preguntas que generó el paciente}

1. En una paciente con preeclampsia moderada (la población), ¿debe indicarse un anticonvulsivante (la intervención) para prevenir las convulsiones (resultado)?

2. En pacientes con preeclampsia (población), ¿qué anticonvulsivante (intervención y comparación) previene con mayor eficacia la convulsión eclámptica? (asumiendo que hubiera beneficio al administrarlo)

\section{Búsqueda bibliográfica}

Se realizó en COCHRANE DATABASE (0n-Line). Se identificó y seleccionó un metaanálisis. Se realizó otra búsqueda en PUBMED . La búsqueda abarcó desde el año 1966 hasta la fecha en idioma inglés. Se utilizaron filtros metodológicos para obtener resultados específicos acerca de estudios sobre terapéutica con la palabras claves (términos MeSH) "preeclampsia", " sulfato de magnesio" y " convulsión". Se identificaron los estudios aleatorizados y metaanálisis. Se encontraron nueve estudios y se seleccionó una revisión sistemática que los incluía.

\section{Preeclampsia y anticonvulsivantes}

La preeclampsia es un desorden multisistémico que afecta a las embarazadas y que se caracteriza por un incremento de la TA y proteinuria. En sus formas severas puede comprometer la función renal, hepática, la coagulación y el sistema nervioso central. También puede provocar insuficiencia placentaria con aumento de la morbimortalidad perinatal. La eclampsia se define como la presencia de una o más convulsiones en asociación con la preeclampsia. Esta patología es la tercera causa de mortalidad materna en nuestro país. Uno de los objetivos del control prenatal es su detección precoz con la intención de prevenir o disminuir la aparición de complicaciones severas incluyendo la convulsión. Con esta finalidad es que se emplean los anticonvulsivantes en mujeres con preeclampsia.

A partir de estudios observacionales y experiencia anecdóticas se comenzó a utilizar el SM para el tratamiento de la preeclampsia y su uso se extendió para la profilaxis de la convulsión en la preeclampsia.

La probabilidad de convulsivar en mujeres con preeclampsia es del $1 \%$. Predecir qué mujeres lo harán es difícil. Por este motivo, las estrategias terapéuticas son heterogéneas. Mientras que en Estados Unidos se estima que el $5 \%$ de las mujeres embarazadas recibe SM antes del parto, en el Reino Unido un $25 \%$ de los obstetras no utiliza ninguna profilaxis anticonvulsivante y sólo el $40 \%$ usa SM.

Los efectos adversos atribuidos al SM son infrecuentes pero de gran relevancia (paro cardíaco o respiratorio). Además se le ha atribuido cierto efecto tocolítico que podría incrementar el riesgo de cesárea, hemorragia post-parto o retención placentaria.

\section{Resumen de los trabajos seleccionados}

1. Duley L, Gulmezoglu AM, Henderson'Smart DJ. Anticonvulsivant for women with preecclamsia. Cochrane database (revisión último semestre de 1999). Nov 1998.

\section{Grupo de trabajo: Cochrane Pregnancy and Child Birth Group.}

Objetivo: evaluar los riesgos y beneficios asociados al uso de anticonvulsivantes en mujeres con preeclampsia.

Fuente de datos: El registro de estudios aleatorizados mantenido y actualizado por el grupo "Cochrane Pregnancy and Child Birth Group". Selección de estudios: se seleccionaron estudios aleatorizados o cuasi aleatorizados que incluyeran mujeres con preeclampsia de cualquier severidad. La intervención estudiada fue la comparación de un anticonvulsivante con placebo (o no anticonvulsivante en el caso de los estudios cuasi aleatorizados) y la comparación de una droga con otra.

Los estudios fueron seleccionados independientemente por dos revisores y las discrepancias resueltas por discusión.

Medición de resultados: para todas las mujeres se empleó como medida principal de resultado la convulsión. En el caso de mujeres aleatorizadas antes del parto, también se midieron las complicaciones del parto y puerperio. Se analizaron resultados de morbimortalidad neonatal. Los resultados se reportaron en riesgo relativo (RR), riesgo atribuible (RA), número necesario para tratar (NNT) y número necesario para " dañar" (NNH).

\section{Resultados}

Anticonvulsivantes vs placebo (o no anticonvulsivante):

Se analizaron cinco estudios (con un total de 1200 mujeres) de los cuales 4 usaron SM como anticonvulsivante. Dichos estudios sugieren una reducción promisoria del riesgo de convulsión eclámptica con drogas (RR 0.35 con IC 95\% 0.13-0.95). Sin embargo el número de eventos fue bajo ( 4 vs 13) y esto se refleja en la amplitud del IC. El RA fue de 0.016 (IC 95\% 0.001 a 0.031 ) y el NNT 63 (IC 95\% 32-1000). El análisis de subgrupos en los trabajos que emplearon SM reveló una reducción no significativa del riesgo de convulsión (RR 0.33 con IC 95\% 0.11-1.01).

La información acerca de otros resultados potencialmente importantes como toxicidad, efectos adversos y resultados perinatales fue escasa.

SM vs fenitoína

EL SM fue mejor que la fenitoína para la prevención de la convulsión eclámptica, pero el número de eventos fue bajo (1 vs 10). El RR fue de 0.05 (IC 95\% 0.00-0.84). El RA fue de 0.009 (IC $95 \% 0.003-0.05$ ) con un NNT de 111 (IC 95\% 67-333).

No se identificó información relevante con relación a la morbilidad materna o perinatal. Sólo se encontró un incremento en el riesgo de cesárea asociada al uso de SM con respecto al uso de fenitoína. El RR fue de 1.21 (IC 95\% 1.05-1.41). El RA fue de 0.048 (IC 95\% 0.0120.084 ) con un NNH de 21 (IC $95 \%$ 12-83).

SM vs diazepam

De los dos estudios realizados no pueden extraerse conclusiones debido al escaso número de pacientes.

Conclusión: existe evidencia insuficiente para establecer riesgos y beneficios del uso de anticonvulsivantes en mujeres con preeclampsia. Por lo tanto no existen claras recomendaciones y hasta nueva eviden- 
cia, la decisión de emplearlos o no deberá basarse en la severidad de la afección y la experiencia previa del médico. En el caso de emplear un anticonvulsivante, la evidencia actual sugiere que el SM es de elección.

2. Witlin AG, Sibiai BM. Magnesium sulfate therapy in preeclamsia and eclamsia. Obstetrics and Gynecology; 92: 883-9.

Objetivo: evaluar la evidencia disponible acerca de la eficacia, los beneficios y riesgos del tratamiento profiláctico anticonvulsivante con SM en mujeres con preeclampsia o eclampsia.

Fuente de datos: Búsqueda en Medline en inglés entre los años 1966 y 1998 utilizando los términos " magnesium sulfate" , " seizure" , " preeclamsia", "eclamsia" e "Hypertension and pregnancy". Se revisó la bibliografía de los artículos recuperados y se realizaron consultas con expertos para referencias adicionales.

Selección de estudios: se efectuó una revisión sistemática seleccionando estudios aleatorizados y controlados, revisiones y estudios retrospectivos que evaluaran eficacia, y efectos adversos del SM en preeclampsia y eclampsia. Se seleccionaron 19 estudios a leatorizados controlados, 5 estudios retrospectivos y 8 reportes observacionales.

Medición de resultados: se reportaron los resultados de cada estudio individual clasificándolos en 3 grupos: aquellos que evaluaron el efecto del SM en eclampsia, aquellos que evaluaron el uso de SM y un antihipertensivo vs no anticonvulsivante y antihipertensivo en preeclampsia severa y aquellos que compararon SM vs fenitoína o placebo. Las medidas de asociación reportadas fueron el Riesgo absoluto y el RR.

Resultados: los 4 estudios aleatorizados y controlados en mujeres con preeclampsia severa revelaron colectivamente una incidencia de convulsiones de $2.8 \%(22 / 793)$ en mujeres tratadas sólo con antihipertensivos y del $0.9 \%(1 / 815)$ en mujeres tratadas con SM y un antihipertensivo. El NNT resultante es 53. Los estudios que evaluaron eclampsia revelaron un $23.1 \%(216 / 935)$ de recurrencia de convulsión en mujeres tratadas con fenitoína o diazepam compara- das con una recurrencia de convulsión del 9.4\% (88/932) en las tratadas con SM.

Conclusión: esta evidencia confirma la eficacia del SM en mujeres con eclampsia y preeclamsia severa. Sin embargo son necesarios nuevos estudios aleatorizados para determinar la eficacia del SM en mujeres con preeclampsia leve o moderada e hipertensión gestacional.

\section{Comentario}

El primer estudio intenta responder si el uso de anticonvulsivantes en mujeres con preeclampsia es mejor que no utilizar tratamiento alguno. Los resultados parecieran demostrar una tendencia favorable hacia la reducción del riesgo de convulsión, pero considerando la amplitud de los IC, estos resultados deben considerarse cautelosamente. En el mejor de los casos, de cada 32 mujeres tratadas, una se beneficiaría con el tratamiento y en el peor escenario varios cientos deberían tratarse para prevenir una convulsión.

Las pacientes incluidas presentaron varios grados de severidad. Por lo tanto, dado que el riesgo de convulsión varía con la severidad; el efecto protector del SM podría estar " diluido" por la incorporación de mujeres con enfermedad leve. En el segundo estudio sólo se incluyeron mujeres graves y se observó una reducción del $69 \%$ en el riesgo de convulsión.

Teniendo en cuenta los trabajos resumidos, es probable que la selección de mujeres con mayor riesgo de convulsión (graves) aumente el impacto clínico de la intervención.

Cabe destacar que en ambos estudios se reporta escasa información acerca de efectos colaterales importantes y no hay información acerca de seguimiento neonatal.

\section{Conclusiones}

Se puede concluir que, con la evidencia hallada, existe insuficiente información para determinar la conducta clínica definida con relación al uso de anticonvulsivantes en mujeres con preeclampsia. La decisión de utilizar o no anticonvulsivantes parecería restringirse a los casos severos. De indicarse, el anticonvulsivante de elección es el SM.

En el caso particular del caso clínico no existe aún suficiente evidencia para indicar SM u otro anticonvulsivante.

Dra. Nora Gimpel

Unidad de Medicina Familiar y Preventiva. Hospital Italiano de Buenos Aires.

Dr. Hugo Krupitzki

Tocoginecólogo. CEMIC 